\title{
Compact Millimeter-Wave Filters Using Distributed Capacitively Loaded CPW Resonators
}

\author{
Farshid Aryanfar, Member, IEEE, and Kamal Sarabandi, Fellow, IEEE
}

\begin{abstract}
In this paper, a method for reducing the size of coplanar-waveguide (CPW) resonators, used in the design of compact bandpass filters, is presented. This is accomplished by incorporating a distributed array of capacitive loads along a resonant CPW line. Design of a miniaturized bandpass filter using capacitively loaded CPW resonators, which are inductively coupled, is presented. Inductively coupled bandpass filters with the proposed capacitively loaded CPW line resonators are designed and a size reduction of $25 \%$, compared with filters using a standard CPW line resonator, is demonstrated. It is shown that, through this size reduction, the insertion loss is minimally increased $(<0.4 \mathrm{~dB})$. A hybrid algorithm, combining the method of moments (MOM) and circuit simulation is used to facilitate design process. Few filters using this technique are designed, fabricated, and measured at $W$-band. An excellent agreement between the simulation and measurement responses of these filters is presented.
\end{abstract}

Index Terms-Bandpass, capacitively loaded, coplanar waveguide (CPW), filter, millimeter wave, miniaturized, $W$-band.

\section{INTRODUCTION}

W ITH THE recent advancement in millimeter-wave devices and new Federal Communications Commission (FCC) regulations making available $10-\mathrm{GHz}$ bandwidth at $W$-band frequencies, gigabit wireless systems providing fiber-like services are fast approaching the commercial market [1]-[3]. Apart from available wide bandwidth, another advantage of millimeter-wave systems is the compact size of the active and passive components; however, in order to achieve on-chip transceiver integration [4] designs, further size reduction of passive elements such as filters is required. This is especially important in the design of high-performance multipole filters, which require high- $Q$ resonators. Filter miniaturization is also important in other applications such as compact millimeter-wave probes and design of filter-lens arrays [5].

One standard way to reduce the size of planar filters is by loading the resonators to create a slow-wave structure. At lower frequencies, there are different techniques to load a resonator, the most recent one is using microelectromechanical systems

Manuscript received July 31, 2005; revised October 11, 2005. This work was supported by the U.S. Army Research Office under Contract DAAD19-00-10115 .

F. Aryanfar was with the Radiation Laboratory, Department of Electrical Engineering and Computer Science, The University of Michigan at Ann Arbor, Ann Arbor, MI 48109 USA. He is now with the Microwave Technologies Research Laboratory, Motorola, Schaumburg, IL 60196 USA (e-mail: faryanfa@gmail.com).

K. Sarabandi is with the Radiation Laboratory, Department of Electrical Engineering and Computer Science, The University of Michigan at Ann Arbor, Ann Arbor, MI 48109 USA (e-mail: saraband@eecs.umich.edu).

Digital Object Identifier 10.1109/TMTT.2005.864109

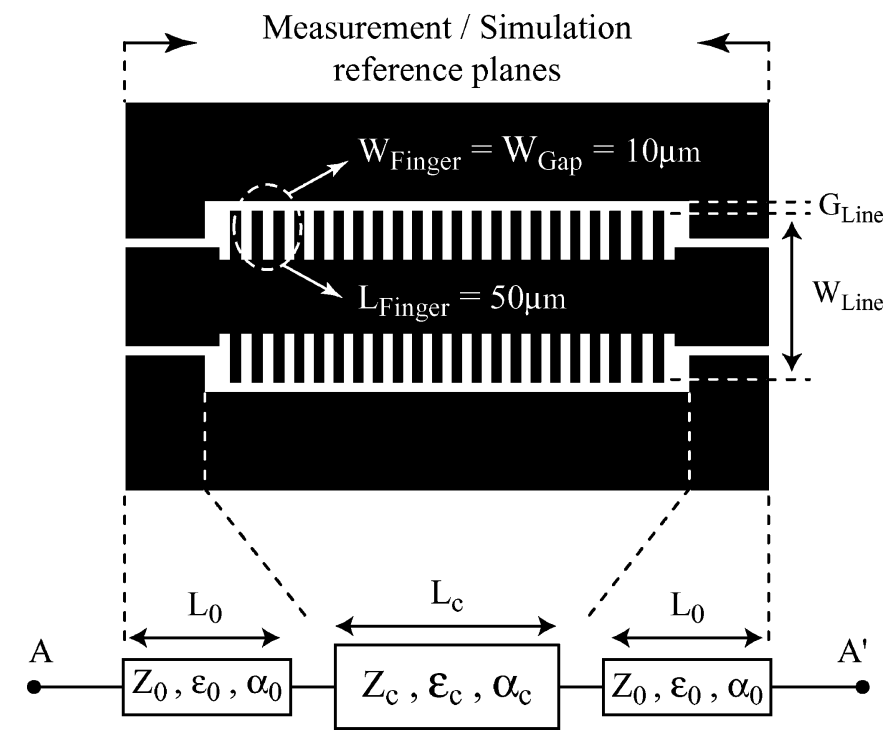

Fig. 1. Capacitively loaded CPW line (type I) and its circuit model used in simulation.

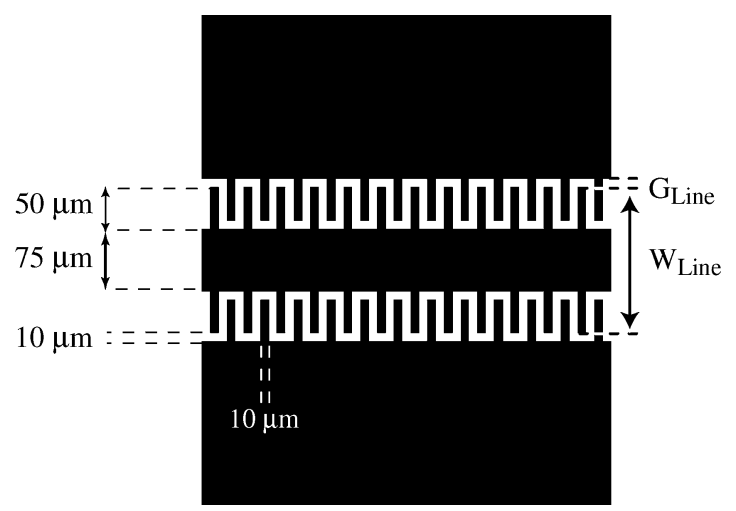

Fig. 2. Capacitively loaded CPW line (type II).

TABLE I

STANDARD AND LOADED CPW LINE DIMENSIONS

\begin{tabular}{lcccc}
\hline & $W_{\text {Line }}$ & $G_{\text {Line }}$ & $W, G_{\text {Finger }}$ & $L_{\text {Finger }}$ \\
\hline Standard CPW & $135 \mu \mathrm{m}$ & $30 \mu \mathrm{m}$ & - & - \\
\hline Loaded CPWs & $175 \mu \mathrm{m}$ & $10 \mu \mathrm{m}$ & $10 \mu \mathrm{m}$ & $50 \mu \mathrm{m}$ \\
\hline
\end{tabular}

(MEMS) bridges along the length resonators to increase the line's capacitance without decreasing the line inductance appreciably [6]. As a result, the electrical length of the resonator, which is proportional to $\beta=\sqrt{L C}$, is increased. However, most parasitic elements associated with three-dimensional (3-D)structures like MEMS bridges, which can be ignored at lower frequencies, have significant effects at millimeter-wave frequencies and will drastically change the filter response. In 


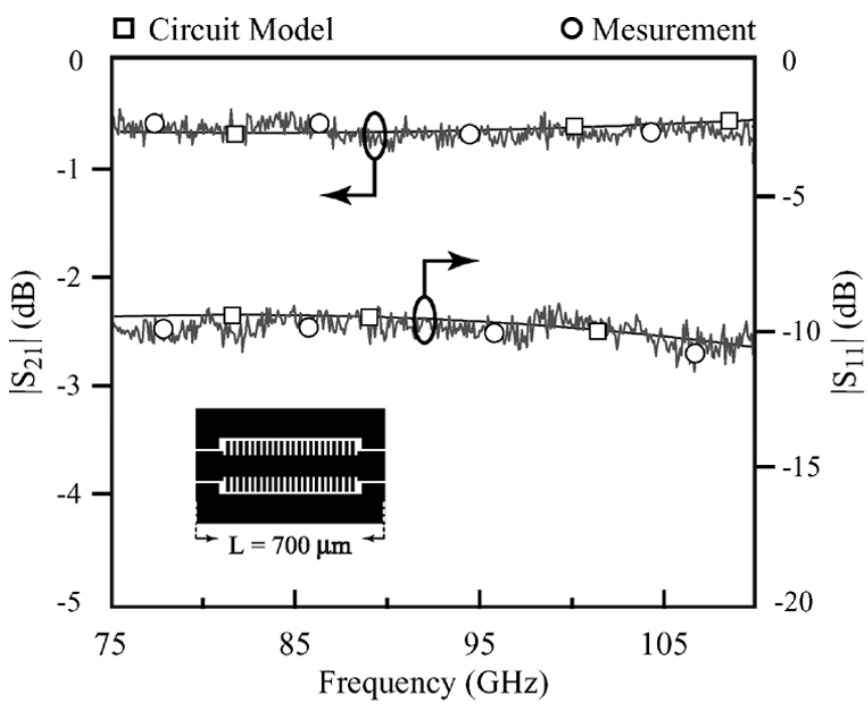

(a)

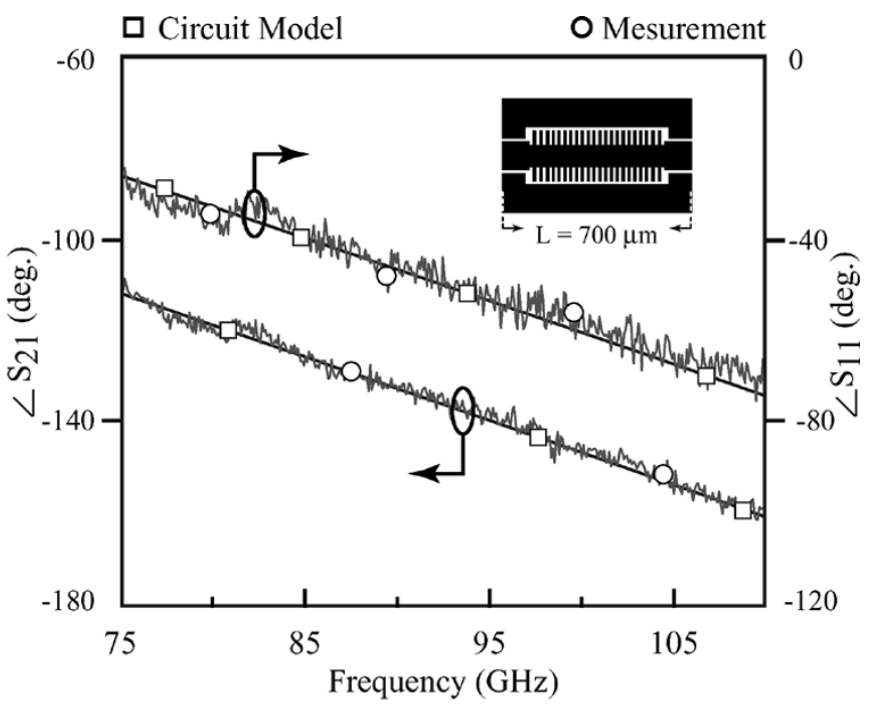

(b)

Fig. 3. Measured and simulated $S$-parameters of capacitively loaded CPW line (type I). (a) Magnitude. (b) Phase.

TABLE II

MEASURED CHARACTERISTICS OF STANDARD AND LOADED CPW LINE @ 95 GHz

\begin{tabular}{cccc}
\hline & $Z_{0}(\Omega)$ & $\beta(\mathrm{rad} / \mathrm{cm})$ & $\alpha(\mathrm{dB} / \mathrm{cm})$ \\
\hline Standard CPW & 70 & 30.49 & 2.1 \\
\hline Loaded CPW (Type I) & 70 & 41.46 & 2.6 \\
\hline Loaded CPW (Type II) & 50 & 51.81 & 3.1 \\
\hline
\end{tabular}

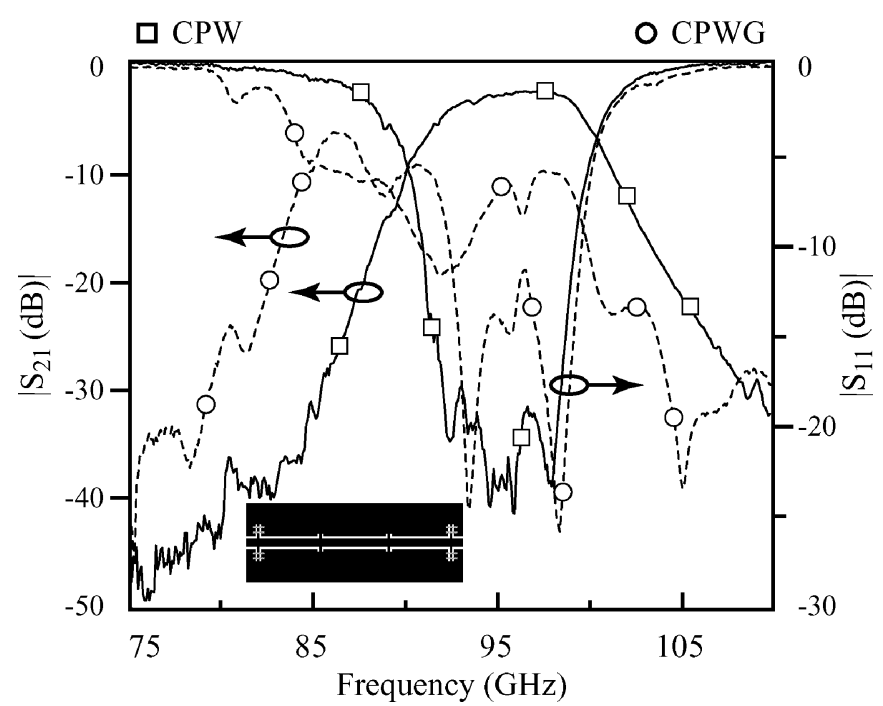

Fig. 4. Effect of probe station's chuck on measured $S$-parameters of a CPW bandpass filter.

addition, incorporation of MEMS bridges is rather cumbersome and costly due to the required complicated fabrication and packaging processes. Hence, to avoid dealing with the parasitic effects and complicated fabrication, use of planar structures with minimal parasitic features are preferred.

Although microwave filters have been studied extensively, the literature concerning planar millimeter-wave filters, especially at $W$-band frequencies, is scarce. One of the major difficulties involved in filter design at millimeter-wave frequencies and above is the absence or inaccuracy of the existing circuit models. This paper provides a hybrid method combining method

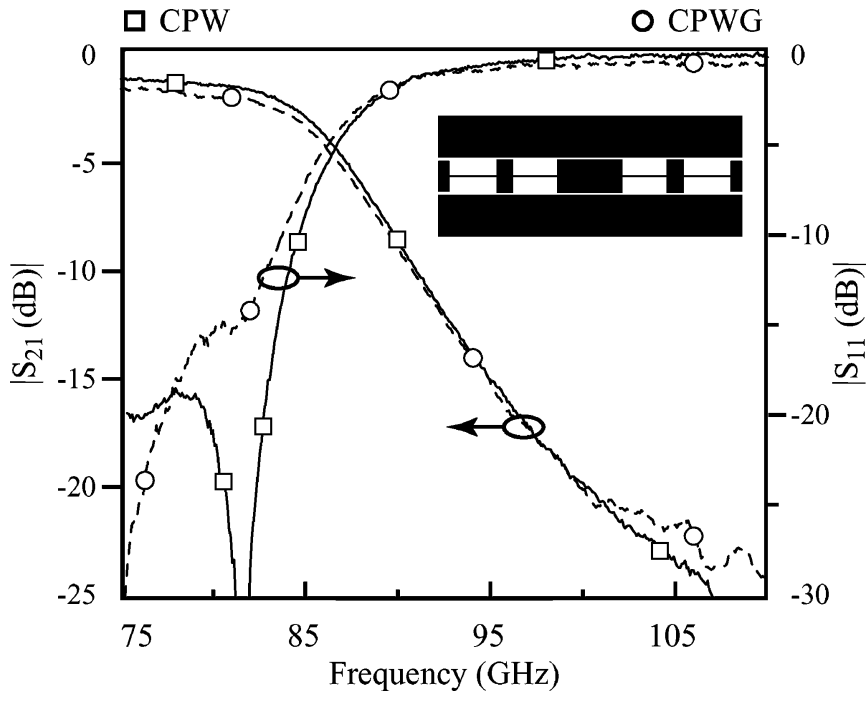

Fig. 5. Effect of probe station's chuck on measured $S$-parameters of a CPW stepped-line low-pass filter.

of moments (MOM) and circuit simulation, which facilitates a systematic approach for filter design at millimeter-waves frequencies. Using this proposed technique and capacitively loaded coplanar waveguide (CPW) resonators, a miniaturized bandpass and a stepped-line low-pass filter are designed and fabricated.

In what follows, the structure of capacitively loaded CPW lines is first introduced and their electromagnetic behavior is characterized. In Section III, sensitivity of CPW circuits to ground effect in traditional on-wafer measurement is shown, and a simple method for avoiding this effect is presented. A hybrid design technique, which is a combination of circuit models and the MOM, is explained in Section IV. Finally, in Section $\mathrm{V}$, using an existing model of shunt inductive stubs for $W$-band frequency range [7] and loaded CPW resonators, design, fabrication, and measurement of a miniaturized bandpass filter is presented and compared with standard CPW line resonators filters. 


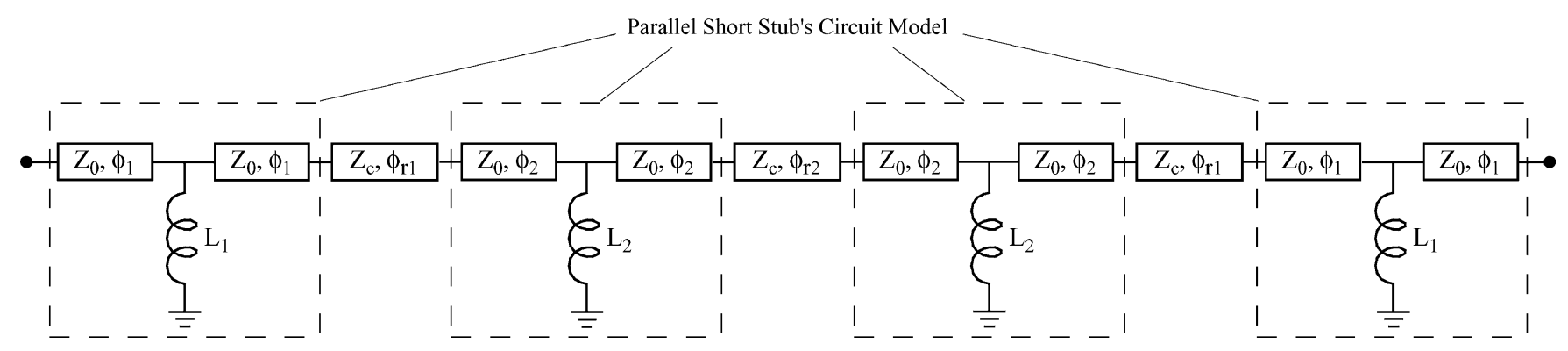

Fig. 6. Circuit model of miniaturized inductive coupled resonator bandpass filter.

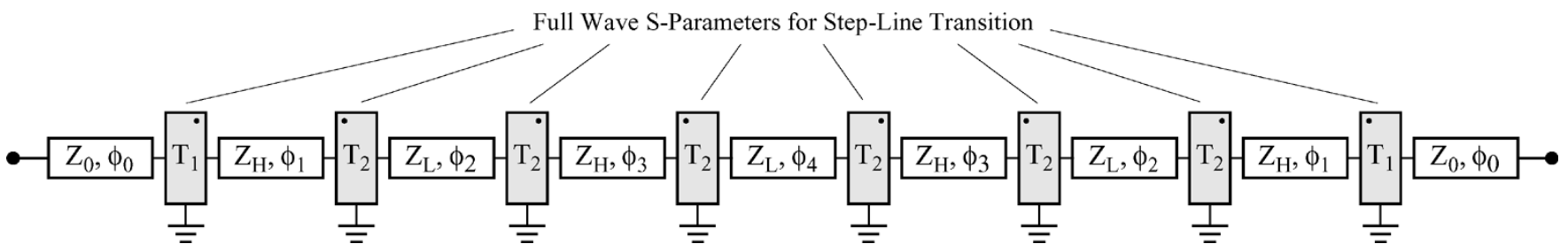

Fig. 7. Hybrid circuit model of a CPW stepped-line low-pass filter.

\section{CAPACITIVELY LOADED CPW LiNES}

In order to increase effective electrical length of CPW resonators and create a slow-wave structure, small parasitic fingers, shown in Figs. 1 and 2, are added to the center conductor and ground of a standard CPW line. For the dimensions shown in Table I, effective electrical lengths of the loaded CPW lines types I and II are increased by $36 \%$ and $70 \%$, respectively. The loaded CPW lines can be modeled as a line with different propagation constants and attenuation coefficients. In order to characterize the electrical properties of the loaded CPW lines, a number of such lines with different lengths were fabricated on a 10-mil-thick $(\simeq 250 \mu \mathrm{m})$ quartz wafer. The measured $S$-parameters, effective line characteristic impedance, effective propagation constant, and attenuation constant are then extracted. Fig. 3 shows the measured and simulated $S$-parameters (magnitude and phase) for the line shown in Fig. 1 with a total length of $700 \mu \mathrm{m}$.

For the dimensions given in Table I, the characteristic impedances, phase constants, and attenuation coefficients of the lines are extracted by comparing the measured and simulated results. These are provided in Table II. As it can be seen, for the loaded CPW line of type I, the electrical length is increased without significant impedance change in comparison with a standard CPW line with similar dimensions. However, the line impedance is decreased for the loaded CPW line of type II because of the increase in the line capacitance caused by periodic inclusion of interdigital capacitors. This provides a higher miniaturization in comparison with the previous case; however, due to the limited quality factor of the interdigital capacitors, it introduces higher attenuation coefficient. Hence, miniaturized filters shown in Section $\mathrm{V}$ are designed using a loaded CPW line of type I only.

The $S$-parameter measurements reported in this paper at $W$-band $(75-110 \mathrm{GHz})$ were obtained using a probe station (for on-wafer measurements), HP-8510C network analyzer, and HP-W85104A millimeter-wave test setup. MOM and circuit simulations are performed using the Agilent Advanced Design System (ADS).

\section{ON-WAFER MEASUREMENT}

The probe station's chuck, which holds the wafers, changes the CPW to a conductor-backed coplanar waveguide grounded (CPWG) line and, thus, it affects the propagation constant of the line [8] and the values of the parasitic elements. In order to eliminate these effects, a supporting structure with a hole in the middle [7] is built and placed on top of the probe station's chuck. Fig. 4 shows significant changes in measured results of a CPW bandpass filter constructed of inductively coupled resonators due to the presence or absence of this test structure.

Placing the filter wafer on the probes station's chuck creates a ground plane for the CPW line, which can not only change the line parameters, but also provides an opportunity for power leakage carried by parallel-plate waveguide modes. However, the cutoff frequency of the first non-TEM parallel-plate mode in this case is around $312 \mathrm{GHz}$ $\left(f_{p}=\left(c / 2 d \sqrt{\epsilon_{r}}, d=250 \mu \mathrm{m}, \epsilon_{r}=3.7\right)\right.$; hence, deformation of the filter response is only due to changes in coupling and the electrical lengths of the resonators. Fig. 5 confirms this postulation by comparing the measured $S$-parameters of a CPW stepped-line low-pass filter once with and once without using this test structure. As it can be seen, there is no significant difference between two cases since stepped-line low-pass filters are not as sensitive to the electrical lengths of the lines as are bandpass filters.

\section{Hybrid DESIGN TeChNIQUe}

The main goal of developing circuit models for various discontinuities is to facilitate the design process and avoid full-wave simulation in the initial design steps because of their time-consuming nature. For this purpose, usually the whole structure is broken into a few parts such that each one can be modeled individually with a minimum or no interaction with others. For example, in the specific bandpass filter selected for miniaturization, which is constructed of inductively coupled CPW resonators, the whole structure can be broken into a number of CPW resonators and inductive stubs. We used an existing 


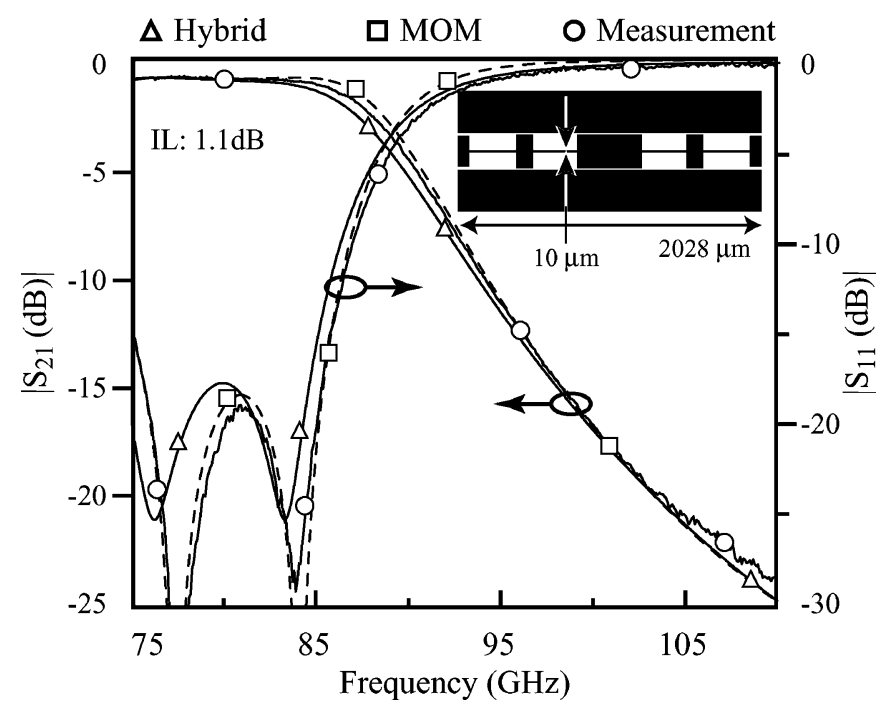

Fig. 8. Simulated and measured $S$-parameters of a CPW stepped-line low-pass filter.

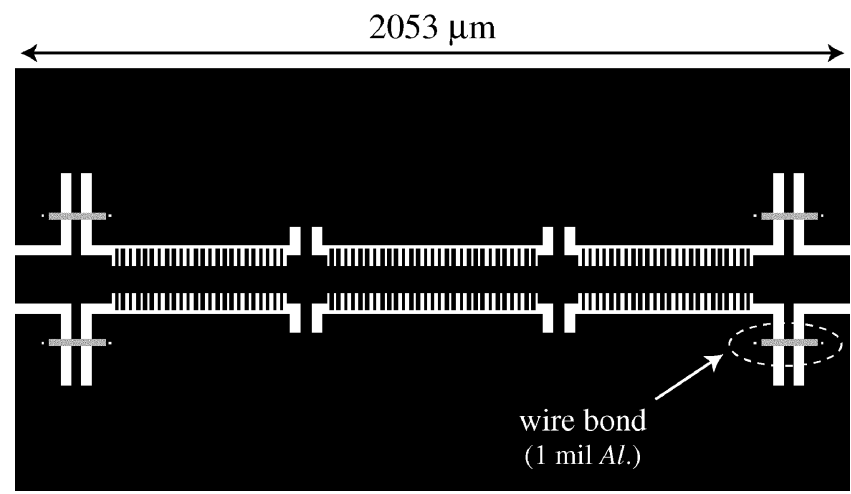

Fig. 9. Layout of miniaturized inductive coupled resonator bandpass filter.

model of shunt inductive stubs for the $W$-band frequency range [7] and transmission-line model, whose parameters are given in Table II. Fig. 6 shows schematics of this filter.

In less complicated cases, where there are only a few types of fixed discontinuities in the whole circuit, it is easier and more accurate to directly use the $S$-parameters extracted from the full-wave simulation in the design process instead of developing a circuit model, which usually needs a significant number of measurements for determining the unknown parameters and validating the circuit model. An example for this case is a CPW stepped-line low-pass filter. As shown in Fig. 7, in a symmetrical case $(N=2 k+1 ; N$ is order of filter), there are only two types of discontinuities encountered in this filter. One between the input line and either a high or low impedance line (T1) and the other between the low and high impedance lines (T2). This filter has been fabricated on a 10-mil-thick $(\simeq 250 \mu \mathrm{m})$ quartz wafer. In Fig. 8, the $S$-parameters obtained from the hybrid simulation are shown versus MoM results and measured values. It can be seen that the hybrid model results have good agreement with the full-wave MOM and measurement results.

\section{Miniaturized BANDPASS Filter}

Here, we demonstrate the miniaturization of a bandpass filter using capacitively loaded CPW line introduced in Section II. A

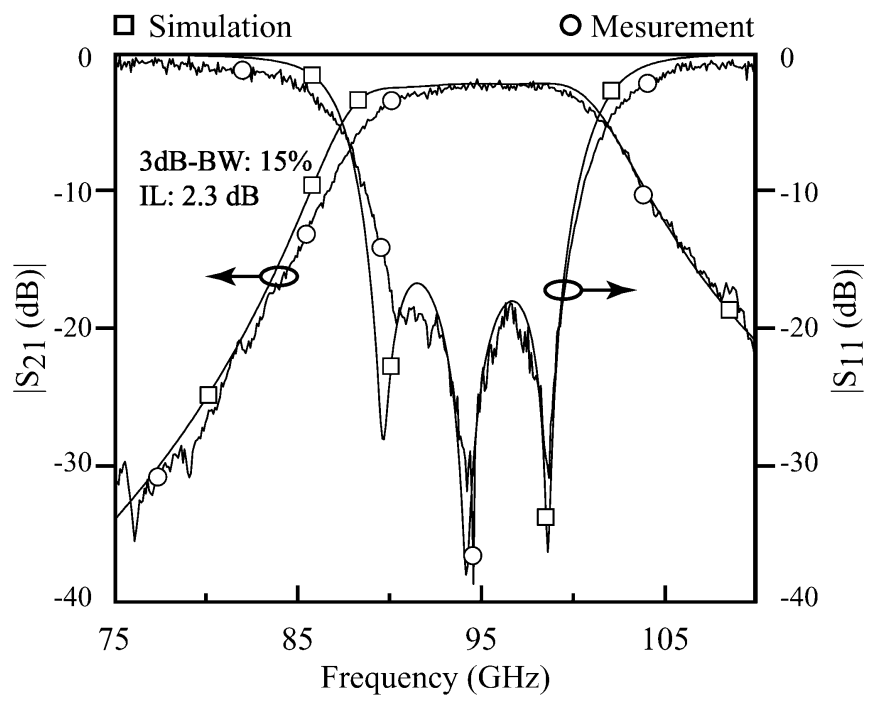

Fig. 10. Measured and simulated $S$-parameters of miniaturized bandpass filter.

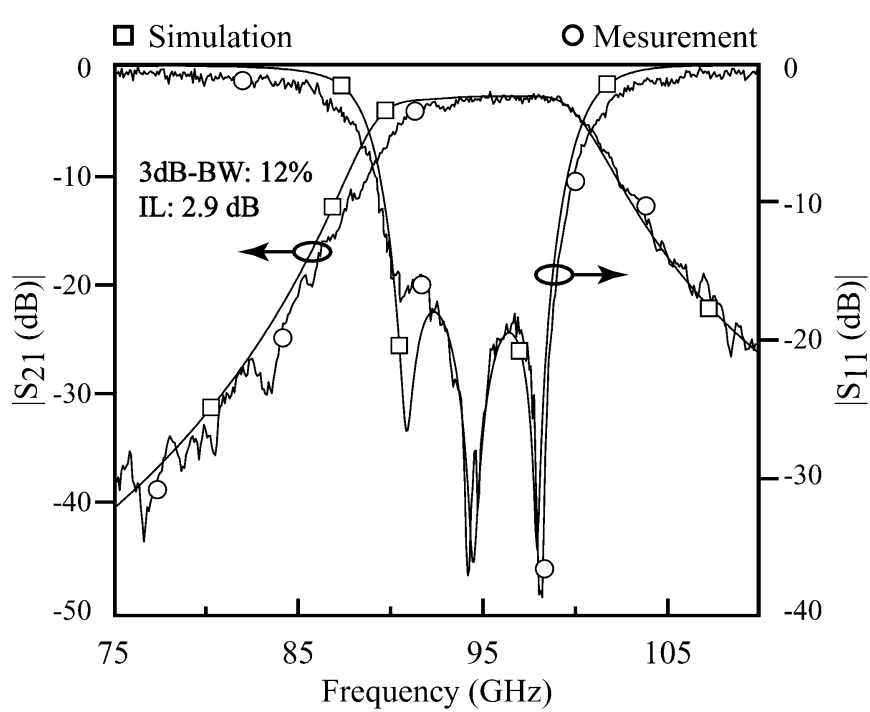

Fig. 11. Measured and simulated $S$-parameters of miniaturized bandpass filter.

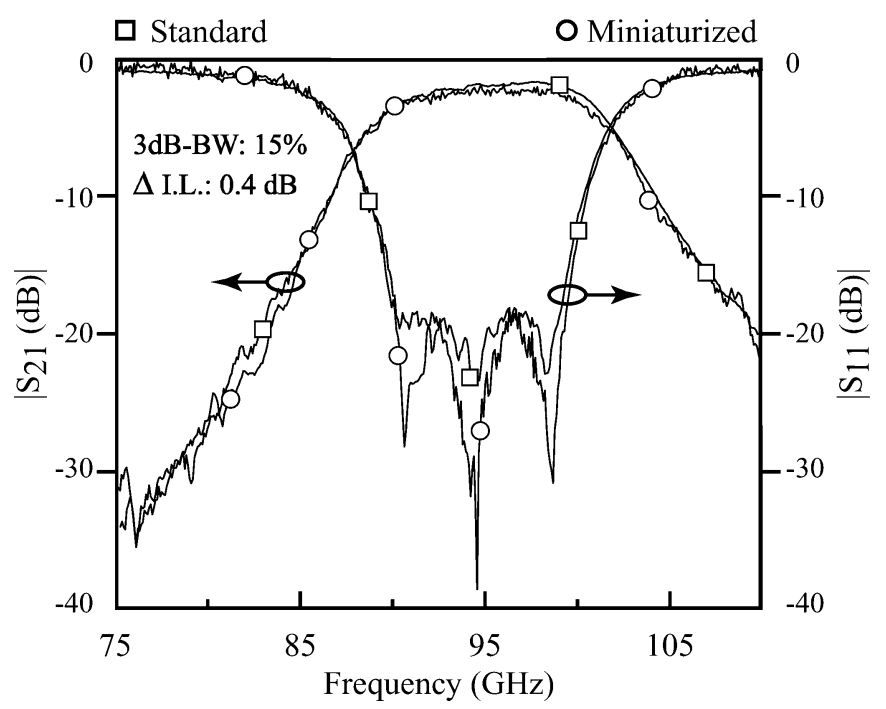

Fig. 12. Comparison of the measured responses of the miniaturized and standard inductively coupled resonator bandpass filters. 
standard inductive coupled resonator bandpass filter [9] is modified by replacing its resonators with capacitively loaded resonators. The schematics and layout of the miniaturized filter are shown in Figs. 6 and 9, respectively. This filter was fabricated on a 10-mil-thick quartz wafer using the standard lithography and wet etching on 3- $\mu \mathrm{m}$ electroplated gold. Figs. 10 and 11 show excellent agreement between the measured and simulated $S$-parameters of this filter for two different designs with different bandwidths. The low-frequency corner of the bandpass filter response in both cases is shown to be slightly degraded due to radiation. This defect can be improved by an appropriate packaging method [10].

Fig. 12 compares the measured results for the miniaturized filter with a standard one. As shown, for a fixed 3-dB bandwidth of $15 \%$, the new filter is slightly $(0.4 \mathrm{~dB})$ lossier than the standard filter, while the size is reduced by $25 \%$.

\section{CONCLUSIONS}

Using slow-wave CPW lines, two planar miniaturized bandpass filters are designed, fabricated, and measured at $W$-band frequencies. For a three-pole filter, $25 \%$ miniaturization has been achieved with a slight increase $(0.4 \mathrm{~dB})$ in the insertion loss. A hybrid technique based on combined circuit and MOM simulation has been presented, which facilitates the design process while maintaining accuracy. The simulation results using the hybrid technique for the miniaturized bandpass filter and a CPW stepped-line low-pass filter has shown excellent agreement with the measured results. Measurement error due to the ground effect of the probe station's chuck through on-wafer measurements of CPW circuits have been studied and a simple solution for eliminating this effect have been introduced.

\section{REFERENCES}

[1] R. Emrick, S. Rockwell, B. Bosco, S. Franson, and J. Holmes, "A 60 GHz transceiver with multi-gigabit data rate capability," in Radio Wireless Conf., Sep. 2004, pp. 135-138.

[2] R. R. Tummala and J. Laskar, "Gigabit wireless: System-on-a-package technology," Proc. IEEE, vol. 92, no. 2, pp. 376-387, Feb. 2004.

[3] M. Ito, S. Kishimoto, K. Ikuina, T. Hashiguchil, K. Ikeda, K. Ohata, K. Maruhashi, and N. Takahash, "1.25 Gbps wireless gigabit ethernet link at $60 \mathrm{GHz}$-band," in IEEE MTT-S Int. Microw. Symp. Dig., vol. 1, Jun. 2003, pp. 373-376.

[4] M. Shen, T. Torrika, W. Michielsen, L. Zheng, X. Duo, and H. Tenhunen, "Design and implementation of system-on-package for radio and mixedsignal applications," High Density Microsyst. Des. Packag. Compon. Failure Anal., vol. 1, pp. 97-104, 2004.

[5] A. Abbaspour-Tamijani, K. Sarabandi, and G. M. Rebeiz, "Antennafilter-antenna arrays as a class of bandpass frequency-selective surfaces," IEEE Trans. Microw. Theory Tech., vol. 52, no. 8, pp. 1781-1789, Aug. 2004.

[6] N. S. Barker and G. M. Rebeiz, "Optimization of distributed MEMS transmission-line phase shifters $U$-band and $W$-band designs," IEEE Trans. Microw. Theory Tech., vol. 48, no. 11, pp. 1957-1966, Nov. 2000.

[7] F. Aryanfar and K. Sarabandi, "Characterization of semi-lumped CPW elements for millimeter-wave filter design," IEEE Trans. Microw. Theory Tech., vol. 53, no. 4, pp. 1288-1293, Apr. 2005.

[8] D. F. Williams, "On-wafer measurement at millimeter wave frequencies," in IEEE MTT-S Int. Microw. Symp. Dig., vol. 3, Jun. 1996, pp. $1683-1686$

[9] J. K. A. Everard and K. K. M. Cheng, "High performance direct coupled bandpass filters on coplanar waveguide," IEEE Trans. Microw. Theory and Tech., vol. 41, no. 9, pp. 1568-1571, Sep. 1993.

[10] S. V. Robertson, L. P. B. Katehi, and G. M. Rebeiz, "Micromachined $W$-band filters," IEEE Trans. Microw. Theory Tech., vol. 44, no. 4, pp. 598-606, Apr. 1996.

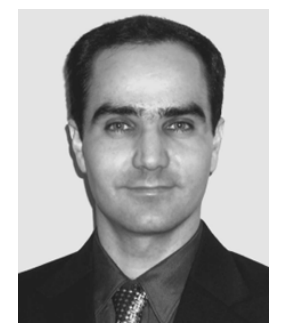

Farshid Aryanfar (S'01-M'05) received the Ph.D. degree in electrical engineering and computer science (with an emphasis on applied electromagnetics and RF circuits) from The University of Michigan at Ann Arbor, in 2005. His doctoral research concerned design and fabrication of a scaled propagation measurement system operating at $W$-band frequencies for wireless channel characterization. His master's thesis concerned wave-propagation modeling in microcellular and picocellular environment using the ray-tracing algorithm, which later led to a 3-D physics-based wave-propagation simulator for wireless channel characterization.

From 1997 to 2000, he was a Senior Microwave Engineer involved with the developing satellite terminals and nondirectional beacon (NDB) transmitters. $\mathrm{He}$ has served as investigator on several research projects sponsored by the Defense Advanced Research Projects Agency (DARPA), the U.S. Air Force (USAF), the U.S. Army, and numerous industries. He is currently with the Microwave Technologies Research Laboratory, Motorola, Schaumburg, IL. His current research interests include microwave/millimeter-wave circuits and systems, wave propagation in complex environment, and wireless channel characterization. He is listed in Who's Who in America.

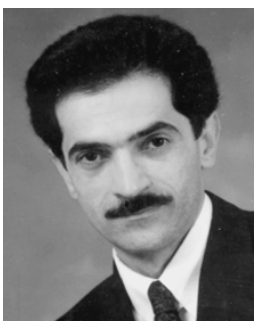

Kamal Sarabandi (S'87-M'90-SM'92-F'00) received the B.S. degree in electrical engineering from the Sharif University of Technology, Tehran, Iran, in 1980, and the M.S.E. and Ph.D. degrees from The University of Michigan at Ann Arbor, in 1986 and 1989, respectively, both in electrical engineering.

$\mathrm{He}$ is currently Director of the Radiation Laboratory and a Professor with the Department of Electrical Engineering and Computer Science, The University of Michigan at Ann Arbor. His research interests include microwave and millimeter-wave radar remote sensing, metamaterials, electromagnetic-wave propagation, and antenna miniaturization. He possesses 20 years of experience with wave propagation in random media, communication channel modeling, microwave sensors, and radar systems and currently leads a large research group including two research scientists, ten Ph.D. students, and two M.S. students. Over the past ten years, he has graduated $22 \mathrm{Ph} . \mathrm{D}$. students. He has served as the Principal Investigator on many projects sponsored by the National Aeronautics and Space Administration (NASA), Jet Propulsion Laboratory (JPL), Army Research Office (ARO), Office of Naval Research (ONR), Army Research Laboratory (ARL), National Science Foundation (NSF), Defense Advanced Research Projects Agency (DARPA), and numerous industries. He has authored or coauthored many book chapters and over 130 papers in refereed journals on EM scattering, random media modeling, wave propagation, antennas, metamaterials, microwave measurement techniques, radar calibration, inverse-scattering problems, and microwave sensors. He has also had over 320 papers and invited presentations in many national and international conferences and symposia on similar subjects. He is listed in American Men and Women of Science, Who's Who in America, and Who's Who in Science and Engineering.

Dr. Sarabandi is a vice president of the IEEE Geoscience and Remote Sensing Society (GRSS), and a member of the IEEE Technical Activities Board Awards Committee. He is the associate editor of the IEEE TRANSACTIONS ON ANTENNAS and PRopagation and the IEEE SENSORS Journal. He is also a member of Commissions F and D of URSI and of The Electromagnetic Academy. He was the recipient of the Henry Russel Award presented by the Regent of The University of Michigan at Ann Arbor (the highest honor The University of Michigan at Ann Arbor bestows on a faculty member at the assistant or associate level), the 1999 GAAC Distinguished Lecturer Award presented by the German Federal Ministry for Education, Science, and Technology given to approximately ten individuals worldwide in all areas of engineering, science, medicine, and law, the 1996 Electrical Engineering and Computer Science Department Teaching Excellence Award, the 2004 College of Engineering Research Excellence Award, the 2005 IEEE Geoscience and Remote Sensing Distinguished Achievement Award, and the 2005 The University of Michigan Faculty Recognition Award. Over the past several years, joint papers presented by his students at a number of symposia [IEEE Antennas and Propagation Society (IEEE AP-S) Symposia (1995, 1997, 2000, 2001, 2003, 2005); the IEEE International Geoscience and Remote Sensing Symposium (1999, 2002); the IEEE Microwave Theory and Techniques Society (IEEE MTT-S) International Microwave Symposium (IMS) (2001), and URSI (2004, 2005)] have been the recipients of Student Prize Paper Awards. 